Annuaire suisse de politique de développement

$17 \mid 1998$

Propriété intellectuelle : quels enjeux pour les pays en développement ?

\title{
6. Politique intérieure et extérieure
}

\section{(2) OpenEdition}

\section{Journals}

Édition électronique

URL : http://journals.openedition.org/aspd/773

DOI : $10.4000 /$ aspd. 773

ISSN : 1663-9669

Éditeur

Institut de hautes études internationales et du développement

Édition imprimée

Date de publication : 1 avril 1998

Pagination : 239-262

ISSN : 1660-5934

Référence électronique

«6. Politique intérieure et extérieure », Annuaire suisse de politique de développement [En ligne], 17|

1998, mis en ligne le 31 juillet 2012, consulté le 08 septembre 2020. URL : http://

journals.openedition.org/aspd/773; DOI : https://doi.org/10.4000/aspd.773 


\section{POLITIQUE INTÉRIEURE ET EXTÉRIEURE}

\subsection{POLITIQUE D'ASILE ET QUESTIONS MIGRATOIRES}

Plusieurs événements importants auront marqué la politique de migration et d'asile de la Suisse en 1997. La décision du Conseil fédéral de renvoyer les ressortissants bosniaques de l'ex-Yougoslavie a provoqué une série de "départs volontaires» dans le le cadre d'un programme d'aide au retour. Cette décision a suscité de fortes réactions de la part des ouvres d'entraide, ainsi que des cantons, dont certains ont refusé d'utiliser les mesures de contrainte pour exécuter les renvois. Pendant l'année 1997, le Parlement a poursuivi la quatrième révision de la loi sur l'asile. La nouvelle loi tient compte de l'évolution des besoins en matière d'admission de réfugiés de guerre et de la violence. Les débats sont très difficiles et la loi ne sera pas adoptée avant 1998. Une commission d'experts en migration (la Commission Hug) a rendu public en août 1997 un rapport dans lequel une nouvelle conception de la politique en matière de migration est proposée; elle englobe l'ensemble des objectifs, instruments et moyens susceptibles d'influencer les migrations et leurs conséquences. Le Conseil fédéral a approuvé les grandes lignes de ce rapport. Enfin, le gouvernement s'est prononcé contre l'initiative des mouvements d'extrême droite visant à limiter à $18 \%$ la population étrangère résidant en Suisse.

\section{$\square$ Demandes d'asile présentées en 1996}

De janvier à décembre 1996,18'000 personnes ont demandé l'asile en Suisse. Le nombre de requérants a augmenté de 5,8\% par rapport à l'année précédente (17'021 personnes). Toutefois ce nombre reste en deçà des chiffres relevés entre 1990 et 1991 , où $35^{\prime} 836$ et 41'629 personnes respectivement avaient déposé une demande d'asile en Suisse'.

Comme toutes ces dernières années, plus d'un tiers des demandes $(34,6 \%)$ étaient le fait de ressortissants de la République fédérale de Yougoslavie (principalement du Kosovar). La part de requérants Bosniaques a diminué, passant de $20,8 \%$ en 1995 à $7 \%$ en 1996. Par contre, la part de ressortissants srilankais a passé de $6 \%$ à $10,6 \%$ du total des demandes. Les autres groupes les plus importants en nombre sont les demandeurs d'asile en provenance de Turquie $(7,3 \%)$, de Somalie et du Zaïre (3,9\%). $87 \%$ des personnes entrées en Suisse sont entrées clandestinement; 744 requérants ont déposé des demandes d'entrée à la frontière et 293 demandes d'entrée ont été remises auprès de représentations suisses à l'étranger.

En 1996, comme durant les années précédentes, le nombre de cas traités par l'Office fédéral des réfugiés (ODR) a dépassé celui des cas nouveaux; le nombre des cas en souffrance a pu ainsi être réduit. Le taux d'acceptation moyen s'est élevé à $12 \%$ en 1996 (14,9\% en 1995 et $12,5 \%$ en 1994).

1. Office fédéral des réfugiés, Statistique en matière d'asile 1996, ODR, janvier 1997. 
Tableau $\mathbf{n}^{0} 15$

Demandes d'asile 1987-1996

\begin{tabular}{lrrrrrrrrrr}
\hline Pays d'origine & $\mathbf{1 9 8 7}$ & $\mathbf{1 9 8 8}$ & $\mathbf{1 9 8 9}$ & $\mathbf{1 9 9 0}$ & $\mathbf{1 9 9 1}$ & $\mathbf{1 9 9 2}$ & $\mathbf{1 9 9 3}$ & $\mathbf{1 9 9 4}$ & $\mathbf{1 9 9 5}$ & $\mathbf{1 9 9 6}$ \\
\hline Afghanistan & 80 & 71 & 134 & 234 & 233 & 278 & 144 & 172 & 114 & 198 \\
\hline Albanie & & & & 250 & 1038 & 546 & 2009 & 50 & 28 & 315 \\
\hline Algérie & & & & & & & 751 & 303 & 388 & 396 \\
\hline Angola & 197 & 322 & 575 & 1134 & 796 & 96 & 542 & 1059 & 493 & 468 \\
\hline Bangladesh & 144 & 195 & 491 & 665 & 593 & 222 & 122 & 286 & 188 & 170 \\
\hline Bosnie-Herzégovine' & & & & & & & 6878 & 3343 & 3534 & 1269 \\
\hline Bulgarie & 8 & 30 & 90 & 681 & 217 & 27 & 25 & 17 & 33 & \\
\hline Chili & 291 & 139 & 39 & 11 & 7 & 6 & 1 & 4 & 0 & \\
\hline Ethiopie & 120 & 131 & 204 & 306 & 394 & 94 & 76 & 100 & 142 & 174 \\
\hline Ex-Yougoslavie & 131 & 818 & 1365 & 6545 & 14205 & 6262 & 5291 & 4124 & 5491 & 6223 \\
\hline Ghana & 101 & 113 & 383 & 450 & 852 & 137 & 66 & 15 & 15 & \\
\hline Inde & 401 & 730 & 720 & 1828 & 886 & 38 & 21 & 48 & 156 & 201 \\
\hline Irak & 27 & 34 & 26 & 71 & 160 & 117 & 79 & 151 & 321 & 413 \\
\hline Iran & 513 & 291 & 211 & 417 & 222 & 140 & 95 & 82 & 110 & 134 \\
\hline Liban & 375 & 529 & 2477 & 5533 & 1352 & 450 & 490 & 170 & 129 & 148 \\
\hline Nigeria & 3 & 8 & 101 & 308 & 515 & 123 & 72 & 115 & 178 & 253 \\
\hline Pakistan & 581 & 659 & 1027 & 1212 & 1339 & 631 & 410 & 420 & 437 & 483 \\
\hline Pologne & 160 & 293 & 255 & 549 & 60 & 64 & 16 & 59 & 3 & \\
\hline Roumanie & 89 & 161 & 431 & 1959 & 2682 & 620 & 113 & 113 & 82 & 70 \\
\hline Somalie & 22 & 22 & 88 & 181 & 910 & 1077 & 2295 & 881 & 478 & 700 \\
\hline Sri Lanka & 895 & 1516 & 4809 & 4774 & 7349 & 2826 & 1744 & 1487 & 1024 & 1965 \\
\hline Syrie & 159 & 89 & 115 & 239 & 179 & 146 & 80 & 44 & 57 & 83 \\
\hline Tchécoslovaquie & 96 & 98 & 171 & 21 & 26 & 25 & 12 & 5 & 1 & 0 \\
\hline Turquie & 5817 & 9673 & 9395 & 7262 & 4324 & 1827 & 1205 & 1068 & 1293 & 1317 \\
\hline Vietnam & 157 & 129 & 168 & 139 & 86 & 69 & 164 & 215 & 159 & 126 \\
\hline Zaïre & 191 & 136 & 419 & 758 & 1426 & 677 & 362 & 276 & 320 & 695 \\
\hline Autres & 267 & 453 & 715 & 1441 & 1731 & 1459 & 1681 & 1359 & 1664 & 2187 \\
\hline Total & & & & & & & & \\
\hline
\end{tabular}

1. Les demandes d'asile des requérants des Etats de Bosnie et d'Herzégovine sont dans les banques de données depuis le début de 1993.

2. A partir de 1993, reste de l'ex-Yougoslavie sans Croatie, ni Slovénie et Macédoine.

Source: DFJP/Office fédéral des réfugiés. 
Tableau n ${ }^{\circ} 16$

Octroi de l'asile et taux moyen de reconnaissance de la qualité de réfugié'

\begin{tabular}{|c|c|c|c|c|}
\hline Nationalité & $\begin{array}{l}\text { De janvier à décen } \\
\text { en chiffres absolus }\end{array}$ & $\begin{array}{l}\text { re } 1966 \\
\text { en \% }\end{array}$ & $\begin{array}{l}\text { De janvier à décembi } \\
\text { en chiffres absolus }\end{array}$ & $\begin{array}{r}\text { re } 1995 \\
\text { en \% }\end{array}$ \\
\hline Bosnie-Herzégovine & 565 & $29.2 \%$ & 902 & $29.4 \%$ \\
\hline Rép. féd. de Yougoslavie & 455 & $7.5 \%$ & 470 & $12.0 \%$ \\
\hline Sri Lanka & 39 & $1.3 \%$ & 26 & $0.6 \%$ \\
\hline Turquie & 621 & $43.8 \%$ & 623 & $49.4 \%$ \\
\hline Irak & 155 & $70.5 \%$ & 84 & $65.6 \%$ \\
\hline Vietnam & 112 & $90.3 \%$ & 141 & $87.6 \%$ \\
\hline Autres & 320 & $5.2 \%$ & 320 & $8.9 \%$ \\
\hline Total/Moyenne & 2267 & $12.0 \%$ & 2648 & $14.9 \%$ \\
\hline
\end{tabular}

1. Taux de reconnaissance $=$ pourcentage des décisions positives par rapport au total des décisions rendues pendant la période d'observation (positives, négatives, non-entrée en matière).

Source: Statistique en matière d'asile 1996, Département fédéral de justice et police, Office fédéral des réfusiés, janvier 1997.

Au total 10'388 requérants d'asile déboutés ont quitté la Suisse ou ont disparu en 1996. Ce nombre est supérieur de 1000 par rapport à 1995.

L'effectif total des personnes entrées dans notre pays comme demandeurs d'asile 2 et y séjournant toujours à fin 1996 était de $130^{\prime} 879^{3}$ (125'448 à fin 1995). En 1996, les dépenses de l'ODR se sont montées à 928 millions de francs ${ }^{4}$ alors qu'en 1995 elles étaient de 821 millions de francs. Ce montant comprend l'argent versé aux requérants et aux réfugiés, les frais administratifs des cantons et diverses dépenses de l'Office fédéral des réfugiés.

En 1990, un arrêté fédéral de portée générale a introduit la notion de "pays d'origine sûr», selon laquelle la Suisse n'entre pas en matière sur des demandes d'asile déposées par des ressortissants de pays où le risque de persécution est «minime». Des exceptions peuvent être faites, si le requérant d'asile fournit des indices qui confirment une persécution individuelle. Cette liste est modifiée sur décision du Conseil fédéral, ajoutant ou enlevant des Etats selon son appréciation. En décembre 1997, la liste contenait les pays suivants: Albanie, Bulgarie, Gambie, Ghana, Inde, Roumanie, Sénégal. Depuis que cette liste existe, le principe en a été repris par quasiment tous les Etats de l'Union européenne.

\section{$\square$ Politique de renvoi et d'aide au retour}

Retour des ressortissants bosniaques en Bosnie-Herzégovine

Au cours de la guerre qui a sévi dans les Balkans, la Suisse a accueilli environ 30’000 personnes chassées de Bosnie par la guerre. A la fin de l'année 1996, environ 25'000 personnes s'y trouvaient encore, en vertu de la loi sur l'asile ou sur les étrangers.

2. Il s'agit de réfugiés reconnus, de personnes admises à titre provisoire, de requérants en cours de procédure, de requérants déboutés en instance d'exécution du renvoi et de détenteurs d'une autorisation de résidence délivrée par la police des étrangers.

3. Ce chiffre cumule depuis des années toutes les personnes ayant passé par la procédure d'asile.

4. Le Courrier, 23 juin 1997 «La grande peur du conseiller fédéral Arnold Koller» (discours du Conseiller fédéral devant l'OSAR). 
Dans ses décisions du printemps 1996, le Conseil fédéral a mis l'accent sur l'encouragement au retour volontaire. L'Office fédéral des réfugiés (ODR) et la Direction pour le développement et la coopération (DDC) ont élaboré un plan d'action applicable jusqu'à la fin de 1996, qui prévoyait l'octroi d'aide à la réintégration des personnes disposées à rentrer, ainsi que l'attribution d'aide en faveur des structures locales dans les régions accueillant les personnes de retour au pays. Le programme fédéral d'aide au retour accordée par la Suisse aux réfugiés de Bosnie-Herzégovine s'est poursuivi tout au long de 1997. Au vu du grand nombre d'inscriptions pour participer au programme d'aide au retour, le Conseil fédéral a accordé 26 millions supplémentaires en janvier 1997. A la mi-octobre 1997 plus de 5000 Bosniaques avaient bénéficié du programme 5 .

En janvier 1997, le Conseil fédéral a réaffirmé l'obligation de rentrer en Bosnie pour les personnes ayant fui la guerre et s'étant réfugiées en Suisse ${ }^{6}$. Cette décision impliquait le retour de 18'000 Bosniaques accueillis en Suisse. Dans un premier temps, les personnes seules et les couples sans enfants, totalisant 8000 Bosniaques, devaient quitter la Suisse jusqu'au 30 avril 1997. En revanche, le délai de départ des familles avec enfants et des mineurs non accompagnés (environ 10 '000 personnes) a été prolongé de huit mois pour arriver à échéance fin avril 1998. Le Conseil fédéral justifie ces départs échelonnés afin de tenir compte des capacités d'accueil de la Bosnie-Herzégovine et de ne pas surcharger les autorités compétentes en matière d'exécution des renvois. Il précise également que les réfugiés reconnus et les personnes titulaires d'une autorisation de séjour délivrée par la police des étrangers peuvent rester en Suisse; il en va de même, provisoirement, pour les déserteurs et les objecteurs de conscience. Enfin, le Conseil fédéral demande aux cantons de prendre en considération des situations particulières (familles d'ethnie mixte ou personnes ayant des problèmes de santé). Sont donc astreintes au retour, pour la période 1997-1998, toutes les personnes chassées de Bosnie par la guerre qui bénéficiaient, durant leur séjour en Suisse, d'une autorisation de courte durée (environ 5000 personnes), celles qui avaient été admises à titre provisoire (10'500), celles dont les procédures d'asile ou de recours connaîtront une issue négative ou celles dont les demandes ont déjà fait l'objet d'un rejet entré en force (3000) ${ }^{7}$.

A la mi-mars, le Conseil fédéral a transmis aux cantons des directives ${ }^{8}$ relatives à la mise en œuvre des arrêtés du Conseil fédéral sur le retour des ressortissants bosniaques. Les cantons ont réagi de manière très différente à la recommandation de la Confédération d'utiliser les mesures de contrainte pour expulser les célibataires et les couples sans enfants qui n'avaient pas quitté le territoire au délai fixé à fin août $1997^{\circ}$. Certains ont mis plus de zèle que d'autres à appliquer la directive. En revanche, le canton de Vaud s'est ouvertement opposé à cette

5. Le programme suisse d'aide au retour comprend à la fois un soutien financier de réinsertion pour les rapatriés et des subventions destinées aux structures locales des communes et des régions qui les accueillent. Ces aspects sont largement décrits dans le chapitre de la coopération au développement, sous la rubrique "Aide aux Etats de l'ex-Yougoslavie», p. 322

6. Confirmation de la décision du Conseil fédéral du 26 juin 1996.

7. Office fédéral des réfugiés, Communiqué de presse, 29 janvier 1997, «Retour en Bosnie des personnes déplacées par la guerre».

8. Département fédéral de justice et police, directive relative à la levée de l'admission collective provisoire de groupes étrangers provenant du territoire de l'ex-Yougoslavie et appartenant à des catégories déterminées du 17 mars 1997.

9. La Liberté, 13 octobre 1997 «Des expulsions se font au compte-gouttes». 
directive; il s'est engagé à étudier chaque situation personnelle avant de prononcer un renvoi. Par ailleurs, il a plaidé pour une véritable politique d'intégration ${ }^{10}$.

L'Organisation suisse d'aide aux réfugiés (OSAR) s'est toujours prononcée en faveur de l'encouragement au retour volontaire des réfugiés de guerre de BosnieHerzégovine. Toutefois, après la décision du Conseil fédéral d'avril 1997 d'obliger 8000 Bosniaques à rentrer dans leur pays, l'OSAR a fait part de ses préoccupations dans un communiqué de presse" et a souligné "que le climat de confiance et de sécurité nécessaire au retour des réfugiés n'était toujours pas réalisé».

Accord de réadmission entre la Suisse et la République fédérale de Yougoslavie Un accord sur la réadmission entre la Suisse et la République fédérale yougoslave a été signé le 3 juillet 1997. Officiellement cet accord permet le rapatriement et la réadmission des ressortissants qui séjournent sur le territoire de l'autre sans y être autorisés. Dans les faits, cette mesure doit permettre à la Suisse d'organiser le retour de 12'500 Yougoslaves ${ }^{12}$ qui ne disposent pas d'autorisation de séjour en Suisse. L'accord, qui précise que les rapatriements doivent se dérouler dans le respect des droits de l'Homme, est entré en vigueur le $1^{\text {er }}$ septembre 1997.

Les œuvres d'entraide ${ }^{13}$ se sont indignées de ce que l'accord soit intervenu à un moment où la situation au Kosovo était en train de se dégrader, les droits de l'Homme ne s'étant nullement améliorés et la répression s'étant renforcée.

Depuis le $1^{\text {er }}$ septembre, date d'entrée en vigueur de l'accord de réadmission, une procédure a été mise en place qui permet à la Suisse de s'assurer que Belgrade garantit aux personnes retournant en ex-Yougoslavie de ne pas être inquiétées. A la fin de décembre 1997, 1000 cas ont fait l'objet d'une procédure complète; l'obligation de départs est énoncée depuis janvier 1998. Une centaine de personnes ont déjà quitté la Suisse en 1997.

\section{$\square$ Révision de la loi sur l'asile}

Amorcée en 1993 en commission d'experts, la révision totale de la loi sur l'asile de 1979, qui devait entrer en vigueur le $1^{\text {er }}$ janvier 1996, n'est pas près d'aboutir. Devant le retard accumulé avant même que ne commencent les délibérations parlementaires, le Conseil fédéral a demandé, en février 1997, de proroger de deux ans, soit jusqu'au 31 décembre 1999, aussi bien l'arrêté fédéral urgent sur la procédure d'asile que l'arrêté fédéral urgent sur les mesures d'économie dans le domaine de l'asile et des étrangers ${ }^{14}$. Le Conseil national a traité de cette question pendant la session d'été, alors que le Conseil des Etats l'a abordée lors de la session d'hiver, en décembre 1997. Vu les divergences entre les deux Chambres, le Conseil national sera amené à traiter à nouveau de la loi lors d'une session en 1998.

10. Le Courrier, 16 avril 1997 «Le canton de Vaud est dubitatif devant les renvois des Bosniaques».

11. Organisation suisse d'aide aux réfugiés, Communiqués de presse du 29 janvier et du 22 mai 1997 concernant le renvoi de réfugiés de guerre en Bosnie-Herzégovine.

12. Ce chiffre comprend, d'une part, les Yougoslaves en situation d'accueil temporaire dont le retour est repoussé de mois en mois et, d'autre part, des travailleurs.

13. Organisation suisse d'aide aux réfugiés, Communiqué de presse, 3 mars 1997.

14. «Message relatif à la prorogation des arrêtés fédéraux urgents sur la procédure d'asile ainsi que sur les mesures d'économies dans le domaine de l'asile et des étrangers", in Feuille fédérale, n 5, vol. I, 11 février 1997. 
Cinq points centraux se dégagent de la révision:

- Création d'une base légale pour l'attribution d'une protection provisoire pour les personnes fuyant des régions en guerre, les «réfugiés de la violence». Ce nouveau statut ôtera aux «réfugiés de la violence» le droit de déposer des demandes d'asile individuelles pendant la période lors de laquelle le Conseil fédéral considère que le pays est en guerre. Le National a étendu la définition aux pays dans lesquels il existe de graves violations des droits de l'Homme, mais il n'a pas été suivi par les Etats.

口 Situation des femmes. Il existe une divergence entre les deux Chambres sur ce point: le National a décidé de ne pas ajouter les violences sexuelles à la liste des préjudices entrant en compte pour l'octroi de l'asile alors que le Conseil des Etats souhaite étendre la protection.

- Amélioration de la procédure. Les députés ont adopté un système de transfert des tâches d'assistance de la Confédération aux cantons. Ces derniers pourront toutefois continuer à faire appel aux œuvres d'entraide.

- Réalisation d'économies dans le domaine de l'assistance. Contre l'avis du Conseil fédéral, le National a par contre refusé de transférer la moitié des charges financières de la Confédération aux cantons pour les personnes ayant une protection temporaire. Berne devra continuer à assumer les frais d'assistance, d'encadrement et d'administration en matière d'asile. Cependant, à l'inverse du National, les sénateurs ont voté à l'unanimité en faveur de l'intégration sociale des étrangers, malgré les dépenses que ce programme entraîne.

- Le Conseil des Etats a durci la répression des abus en matière d'asile et a décidé que les étrangers qui sont entrés clandestinement en Suisse ne pouront plus déposer de demandes d'asile.

La révision totale de la loi sur l'asile est extrêmement complexe de par le grand nombre d'articles sujets à discussion (117 au total) et de par la diversité des aspects abordés. En l'état actuel du débat il semble que la loi sur l'asile va se durcir; cependant il faudra attendre qu'elle soit sous toit, vraisemblablement en 1998, pour se faire une idée plus précise de la nouvelle orientation. Les débats aux Chambres sont très marqués par la menace d'un référendum de l'UDC. Les milieux de l'asile sont partagés sur l'évolution des débats; certains fustigent le très net durcissement de la législation en vigueur; quant à l'OSAR, elle se refuse à préjuger de l'issue des débats parlementaires ${ }^{15}$.

\section{$\square$ Politique de migration (rapport Hug)}

Un rapport intitulé Nouvelle conception de la politique en matière de migration a paru en août $1997^{16}$. Mandatée par le Conseil fédéral en 1996, la commission d'experts présidée par l'ancien directeur de l'OFIAMT, Klaus Hug, a été chargée

15. Journal de Genève, 12 décembre 1997 «Asile, un parlement sous influence». Pour connaître la position détaillée des œuvres d'entraide sur la révision de la loi sur l'asile, deux documents peuvent être consultés: Organisation suisse d'aide aux réfugiés, Révision totale sur l'asile - Projet commenté, OSAR, juillet 1997. Justice et Paix, Pour une vraie protection provisoire - Réflexion dans le cadre de la révision totale de la loi sur l'asile, Jeau-Claude Huot, J + P Texte $1 / 97$.

16. Office fédéral des réfugiés, Une nouvelle conception de la politique en matière de migration, rapport de la Commission d'experts en migration par ordre du Conseil fédéral, Berne, août 1997. A commander à l'ODR, Taubenstrasse 16, 3003 Berne, tél. 031/325.96.07, fax 031/324.80.47. 
de rédiger des propositions concrètes concernant une future politique migratoire. La commission a présenté son rapport au Conseil fédéral en août 1997. Les grandes lignes de la politique d'accueil des étrangers ne se trouvent pas vraiment modifiées. Toutefois, ce rapport suscite l'intérêt parce qu'il met en doute un certain nombre de concepts qui ont porté la politique suisse en la matière, notamment le modèle des trois cercles. Par ailleurs, il propose de s'engager plus fortement dans une politique non discriminatoire et pour une intégration effective de la population étrangère.

La Suisse fait partie des pays occidentaux qui accueillent le plus d'immigrants proportionnellement à son chiffre de population. En 1996, la population étrangère résidante constituait un cinquième de la population dans son ensemble. Il est apparu qu'une nouvelle loi sur les étrangers, devant régler de façon cohérente toutes les questions liées à l'admission, l'intégration et le renvoi des migrants, s'avère nécessaire.

La commission part du principe que la pression migratoire que connait la Suisse continuera à s'accentuer. Elle constate également que la demande de maind'œuvre bien formée ira en augmentant et projette que les problèmes que l'intégration pose à la société iront en empirant. Cette évolution se fera parallèlement à la poursuite du processus européen d'intégration. Et de constater que dans ce contexte, les mesures de politique migratoire doivent être adaptées à ces nouvelles donnes. Dans son rapport, la commission définit la migration comme «un transfert passager ou durable du domicile au-delà des frontières»; le rapport n'opère pas de distinction entre les requérants d'asile et les autres étrangers.

\section{Buts et mesures de la nouvelle politique en matière de migration}

Les mesures proposées par la commission concernent les domaines de la politique d'admission, d'intégration, du départ, du renvoi et de la politique migratoire extérieure. La mise sur pied de structures adéquates et d'une politique d'information sur des thèmes liés aux questions de la migration est également nécessaire.

\section{- Politique d'admission}

Le rapport relève que la politique d'admission de main-d'œuvre étrangère doit servir les intérêts globaux de l'économie, tout en tenant compte des besoins culturels et scientifiques de la Suisse. Les possibilités professionnelles d'intégration à long terme doivent être considérées lors de l'admission en vue d'un séjour permanent. Il est relevé que les étrangers doivent être admis en Suisse lorsque des obligations de droit international ou des raisons humanitaires le requièrent.

L'abandon du modèle des trois cercles constitue l'une des principales propositions concernant la politique d'admission. Introduit en 1991, le modèle des trois cercles accorde la priorité aux ressortissants des pays de l'UE et de l'AELE. En deuxième lieu viennent les Etats-Unis et le Canada. Du cercle extérieur (le reste du monde), par contre, ne doivent être admis que les travailleurs qualifiés dont notre économie a besoin. Le modèle des trois cercles n'est pas formellement ancré dans une loi mais résulte d'une pratique administrative approuvée par le Conseil fédéral.

Une deuxième proposition relève que l'admission des ressortissants de l'Union européenne se fera conformément à l'accord bilatéral prévu avec l'UE sur la circulation des personnes. Accord qu'il conviendrait d'étendre aux Etats de 
l'AELE. Par contre, le contingentement est maintenu pour l'admission de la main-d'œuvre en provenance d'Etats non membres de l'UE, et cette maind'œuvre devra être hautement qualifiée. Troisièmement, pour estimer avec précision la capacité d'intégration professionnelle des migrants un système de points sera introduit; il permettra d'évaluer la formation, l'expérience et la capacité d'adaptation professionnelle ainsi que les connaissances linguistiques. Enfin, le statut des différents modes de séjour est simplifié; le statut des saisonniers est aboli.

\section{- Politique d'intégration}

La nouvelle politique vise à une meilleure intégration que ce n'est le cas actuellement. Les mesures proposent de faire bénéficier la population étrangère résidante des mêmes possibilités que les nationaux dans les domaines scolaire, professionnel et social. La naturalisation sera facilitée pour des étrangers assimilables à des indigènes de par leur naissance ou de par leur séjour durable en Suisse.

\section{- Politique du renvoi}

Les décisions de renvoi devront être exécutées de manière systématique. Les dispositions pénales déjà en vigueur contre les activités des passeurs, la présence illégale en Suisse ou le travail au noir (employeurs et employés) devront s'appliquer rigoureusement.

\section{- Politique migratoire extérieure}

Sur le plan international, la Suisse contribue à lutter contre les «migrations forcées». Pour ce faire, elle cherche à soutenir les pays à haut potentiel migratoire en les encourageant au respect des droits de l'homme et de la démocratie. De même, il est recommandé de tenir davantage compte, en matière d'aide au développement, des pays à haut potentiel migratoire.

La commission rejette l'idée d'une loi sur la migration au sens d'une loi-cadre chapeautant la loi sur le séjour et l'établissement des étrangers (LSEE) et la loi sur l'asile. Elle rappelle que cette dernière fait l'objet d'une révision totale qui devrait aboutir en 1998 et affirme qu'une révision totale de la LSEE s'impose également.

En octobre 1997, le Conseil fédéral a constaté «que les conclusions et les propositions de la commission correspondent fondamentalement aux objectifs qu'il s'est fixés pour la présente législature. Il est aussi d'avis que les travaux législatifs doivent se concentrer sur une révision totale de la loi sur le séjour et l'établissement des étrangers (LSEE)» ${ }^{17}$. Le Conseil fédéral a, jusqu'ici, toujours défendu l'idée que le modèle des trois cercles était conforme tant à la Constitution fédérale qu'aux obligations qui incombent à la Suisse en vertu du droit international. Mais il reconnaît que «depuis l'introduction de ce modèle, en 1991, l'expérience a cependant montré que le deuxième cercle (principalement EtatsUnis et Canada) revêt une importance numériquement moindre qu'escompté». Dans ces conditions, il approuve le modèle d'admission se référant aux contingents, renonçant au modèle des trois cercles. Toutefois, il se montre «sceptique» quant à la mise en œuvre d'un système de points. Le Conseil fédéral transmet au Parlement le rapport de la commission d'experts Hug, ainsi que sa position.

17. Département fédéral de justice et police, Communiqué de presse, 30 octobre 1997, «Migration: le Conseil fédéral définit la marche à suivre». 


\section{$\square$ Initiative pour une réglementation de l'immigration}

En août 1995, une initiative populaire "pour une réglementation de l'immigration» a été déposée. Cette initiative préconise l'inscription dans la Constitution fédérale d'un taux maximum de $18 \%$ de la part de la population étrangère en Suisse. Munie de 121'000 signatures, elle provient des milieux d'extrême droite, dont le comité de soutien est composé de politiciens de l'UDC, du Parti de la liberté et des Démocrates suisses. Pour ne pas dépasser le quota de $18 \%$, les auteurs de l'initiative proposent une classification des étrangers. Dans une première catégorie seraient considérés les scientifiques hautement qualifiés, les cadres et les étudiants qui seraient exclus du quota de $18 \%$. Par contre, les requérants d'asile, les personnes admises provisoirement et les personnes déplacées par la guerre séjournant depuis plus d'une année en Suisse seraient comptabilisées. En cas de dépassement des $18 \%$, des départs volontaires seraient encouragés et l'octroi de permis de séjour gelé. De plus l'initiative réclame une réglementation plus stricte à l'intention des requérants d'asile et propose de supprimer l'assistance financière susceptible de favoriser l'attrait de la Suisse.

Dans son message rendu public en août 1997, le Conseil fédéral rejette cette initiative, sans présenter de contre-projet ${ }^{18}$. Pour le Conseil fédéral l'acceptation de cette initiative aggraverait l'isolement de la Suisse. Elle mettrait en péril l'accord international avec l'Union européenne sur la libre circulation des personnes, ainsi que la marge de manœuvre de l'économie suisse en matière de maind'œuvre. En outre le renforcement des conditions d'admission risquerait de provoquer des mesures de rétorsion à l'égard des ressortissants suisses. Finalement le Conseil fédéral relève que cette initiative va à l'encontre de plusieurs de ses objectifs majeurs, dont l'intégration des étrangers, la circulation des personnes, l'accueil des réfugiés. Le peuple sera appelé à se prononcer sur cette initiative.

\section{SOURCES}

«Message concernant l'initiative populaire 'pour une réglementation de l'immigration'», in Feuille fédérale, n³9, vol IV, 7 octobre 1997.

«Retour des ressortissants bosniaques en Bosnie-Herzégovine», in Feuille fédérale, n 14, vol. II, 15 avril 1997.

Office fédéral des réfugiés, Statistique en matière d'asile 1996, ODR, janvier 1997.

Office fédéral des réfugiés, Communiqués de presse parus en 1997.

Office fédéral des réfugiés, Une nouvelle conception de la politique en matière de migration, rapport de la Commission d'experts en migration par ordre du Conseil fédéral, Berne, août 1997.

Asylon, revue de l'Office fédéral des réfugiés, parution de 1997.

Fulvio Caccia, in La Vie économique, «Intégration de la population étrangère - Plaidoyer pour une prise en compte judicieuse», 11/97.

Organisation suisse d'aide aux réfugiés, Soixante ans d'aide aux réfugiés 1936-1996, OSAR, 1996.

Organisation suisse d'aide aux réfugiés, Révision totale sur l'asile - Projet commenté, OSAR, juillet 1997.

Justice et Paix, Pour une vraie protection provisoire - Réflexion dans le cadre de la révision totale de la loi sur l'asile, Jean-Claude Huot, J + P Texte 1/97.

Vivre ensemble, bulletin de liaison pour la défense du droit d'asile, $\mathrm{n}^{\text {os }} 61$ à 66, parution de 1997.

Le Courrier, 21 décembre 1997 «Le Conseil des Etats traque l'abus et tue l'asile».

Journal de Genève, 18 juin 1997 «La révision de la loi sur l'asile votée par le Conseil national fait beaucoup de mécontents», 3 juillet 1997 «Avec tant d'étrangers au chômage, il faut repenser la politique migratoire suisse», 21 août 1997 «Le Conseil fédéral rejette l'initiative visant à limiter à 18\% le nombre d'étrangers», 30 août 1997 «Les experts du Conseil fédéral préconisent une refonte totale de la politique d'immigration», 31 octobre 1997 «Berne veut remplacer la politique des trois cercles par un nouveau système», 12 décembre 1997 «Pour J.-D. Gerber, l'ODR n'est pas une machine à expulser les requérants d'asile», 20 décembre 1997 «Grande perplexité autour de la révision de la loi sur l'asile».

18. «Message concernant l'initiative populaire 'pour une réglementation de l'immigration'», in Feuille fédérale, n³9, vol. IV, 7 octobre 1997. 
La Liberté, 18 juin 1997 «Le Conseil national est favorable à la cantonalisation de l'assistance», 21 août 1997 «L'initiative Aubry fixe à 18\% le taux d'étrangers, Koller dit non», 30 août 1997 «Les experts du Conseil fédéral liquident la théorie des trois cercles», 21 décembre 1997 «Asile, une série de divergences».

Nouveau Quotidien, 18 juin 1997 «La Suisse se barricade par tous les moyens contre les requérants d'asile», 31 août 1997 «Le Conseil fédéral décloisonne l'immigration», 1 1 er septembre 1997 «Le Conseil fédéral peut justifier l'abandon de la politique des trois cercles».

Neue Zürcher Zeitung, 6. Juni 1997 «Harzige Asylrechtsrevision im Nationalrat», 14. Juni 1997 «Asylpolitische Gewitterwolken und Lichtblicke», 18. Juni 1997 «Heftige Kontroverse un Arbeitlosenversicherung», 30. August 1997 «Vorschläge für kohärente Migrationspolitik», 15. November 1997 «Klärung der Interessen in der Ausländerpolitik», 29. November 1997 «Von drei zu zwei Kreisen?», 12. und 19. Dezember 1997 (Asylgesetzrevision im Ständerat).

\section{SITES INTERNET}

Site du Haut-Commissariat des Nations Unies pour les réfugiés: http://www. unhcr. ch/

Le journal Le Courrier a créé un site comptant plus de 300 articles parus depuis 1994 concernant la politique d'asile en Suisse: http://www. imaginer. ch/courrier/refugies/

\subsection{BLANCHIMENT D'ARGENT - FUITE DE CAPITAUX - CORRUPTION}

La lutte contre le blanchiment d'argent comme celle contre la corruption s'organisent tant au niveau international que sur le plan national. La Suisse n'est pas épargnée par certaines affaires de corruption impliquant des entreprises ou des fonctionnaires suisses. Les fonds de dictateurs déposés dans les banques helvétiques ternissent l'image de la place financière. Enfin, la crainte de voir des pans de l'économie passer aux mains du crime organisé se fait plus précise. Si, dans un premier temps, les critères éthiques motivaient la lutte contre la criminalité en col blanc, un certain nombre d'études ont démontré les effets économiques néfastes que causaient de tels délits. Ainsi la lutte contre ce fléau a pris, depuis quelques années, une dimension nouvelle. En 1997, des mesures contraignantes ont été prises au niveau international (convention de l'OCDE, engagements du FMI et de la Banque mondiale, Déclaration de Lima contre la corruption), qui sont autant de signes de la volonté politique d'en finir avec des pratiques douteuses. Les discussions internationales permettent d'alimenter et d'influencer le débat au niveau suisse. Durant l'année passée sous revue, la loi d'entraide judiciaire internationale est entrée en vigueur, ce qui a permis de débloquer quelques cas, et la loi sur le blanchiment d'argent a été adoptée par les Chambres.

\section{$\square$ Loi sur le blanchiment d'argent dans le secteur financier}

La loi concernant la lutte contre le blanchiment de l'argent dans le secteur financier (LBA) ${ }^{19}$ a été discutée lors des sessions parlementaires de 1997 et adoptée par les Chambres fédérales lors de la session d'automne. La nouvelle loi sur le blanchiment d'argent fait partie du renforcement de l'arsenal législatif souhaité par le Conseil fédéral et le Parlement afin de mieux combattre les nouvelles formes de criminalité. Elle vise à compléter les mesures pénales entrées en vigueur en 1990 et 1994.

ASTM 1997, p. 99.

19. Loi sur le blanchiment d'argent du 10 octobre 1997, in Feuille fédérale, n 41 , vol. IV, 21 octobre 1997. 
Dans le message relatif à la loi sur le blanchiment d'argent, le Conseil fédéral rappelle que le blanchiment concerne l'argent généré par le crime organisé. Les organisations criminelles accumulent de grandes quantités d'argent par des activités de commerce illégal (de la drogue, des armes, des femmes et enfants, de la pornographie) ou par des délits financiers à grande échelle (vol, fraude, chantage, détournement de fonds). Dans le blanchiment d'argent, deux phases sont à distinguer. Au cours de la première, il s'agit de convertir les valeurs patrimoniales illégales en effets négociables, puis, au cours de la seconde, de les introduire dans le circuit économique ${ }^{20}$.

La loi sur le blanchiment d'argent constitue une étape importante dans l'évolution de la législation financière. La nouvelle loi introduit deux innovations importantes. La première consiste à étendre l'obligation de diligence à l'ensemble des intermédiaires financiers. Elle s'adresse non seulement aux banquiers et aux assureurs, mais aussi à tous les intermédiaires financiers (gérants de fortune n'étant pas assujettis à la surveillance des banques, notaires, fiduciaires, avocats). Les intermédiaires doivent en particulier vérifier l'identité de la personne avec laquelle ils sont en relation et s'assurer de l'identité de l'ayant droit économique, une mesure destinée à éviter les hommes de paille. La deuxième nouveauté réside dans l'obligation de communication et de blocage de fonds en cas de soupçon de blanchiment d'argent. L'annonce est effectuée auprès du Bureau de communication en matière de blanchiment d'argent, géré par l'Office central de lutte contre le crime organisé. Les fonds restent alors bloqués cinq jours, pendant lesquels le bureau doit rendre une décision, faute de quoi la relation d'affaires peut suivre son cours. C'est le bureau qui dénonce les cas de blanchiment à l'autorité pénale. Afin de retrouver la trace des transactions réalisées avec des capitaux douteux, des documents doivent être établis pour chaque transaction (le délai de conservation des documents a été établi à dix ans). Enfin, les intermédiaires qui enfreignent l'obligation de communiquer pourront se voir infliger une amende de 200'000 francs au plus. La loi est fondée sur le principe de l'autorégulation: les groupements d'intermédiaires ont la possibilité de créer des organismes d'autorégulation édictant des règlements garantissant le respect des obligations prévues dans la loi. Il s'agit principalement d'étendre la Convention de diligence des banques à l'ensemble du secteur financier.

Peu de divergences sont apparues lors des débats entre les deux Chambres. Le point le plus sensible en discussion concernait la distinction entre le blanchiment intentionnel et celui par négligence. Le Conseil national avait décidé de préciser dans la loi que la violation de l'obligation de dénoncer à l'autorité les cas susceptibles de donner lieu à un blanchiment était punissable, que l'infraction soit intentionnelle ou due à une négligence. Le Conseil des Etats ne souhaitait punir que les infractions intentionnelles, craignant de trop nombreuses annonces non fondées. Finalement, les sénateurs ont suivi une proposition de compromis suggérant de supprimer la référence aux notions d'intention et de négligence dans la LBA, mais de suivre le Code pénal selon lequel la négligence est punissable. Une autre divergence a soulevé de nombreux débats lorsqu'il s'agissait de déterminer s'il y avait obligation ou possibilité de dénoncer les soupçons de blanchiment.

20. «Message relatif à la loi fédérale concernant la lutte contre le blanchissage d'argent dans le secteur financier», in Feuille fédérale, $\mathrm{n}^{\circ} 37$, vol. III, 17 septembre 1996. 
Les milieux économiques et les partis de droite souhaitaient que soit reconnue la possibilité de dénoncer; à l'inverse, les praticiens de la lutte contre le blanchiment et la gauche étaient en faveur d'une obligation, notion qui a été retenue. La LBA doit entrer en vigueur au cours du premier semestre 1998.

\section{$\square$ Activité du Groupe d'action financière de l'OCDE (GAFI)}

La Suisse participe activement aux travaux du Groupe d'action financière sur le blanchiment de capitaux de l'OCDE. Le GAFI a été créé lors du Sommet du G7 qui s'est tenu à Paris en 1989, avec pour mission d'étudier des mesures destinées à combattre le blanchiment de capitaux. En avril 1990, il a publié un rapport de «quarante recommandations» afin de lutter contre ce délit. Ces dernières ont fait l'objet d'une première révision en 1996 et sont maintenant devenues des normes largement reconnues. Toutefois, le GAFI considère que des domaines importants méritent des études et des directives supplémentaires, travaux auxquels il s'attelle. Le GAFI regroupe 26 Etats $^{21}$ et deux organisations régionales ${ }^{22}$ représentant les principaux centres financiers mondiaux.

Dans son Rapport annuel 1996-1997²3, le GAFI constate que des progrès sont accomplis dans l'application des «quarante recommandations» pour lutter contre le blanchiment de capitaux, tant au sein qu'en dehors de la zone GAFI. Par un système d'évaluation mutuelle, le GAFI consacre une part importante de son travail au suivi de l'application des recommandations par ses membres (les résumés des évaluations sont présentés dans le Rapport annuel). Comme tous les autres membres du GAFI, la Suisse a fait l'objet d'une analyse en 1997-1998 dans le cadre du deuxième cycle d'évaluation mutuelle. Les conclusions de l'étude seront publiées dans le rapport 1998 du GAFI, à paraître en juin.

L'une des tâches importantes menées à bien en 1996-97 consiste en l'examen des tendances et des techniques de blanchiment de capitaux liées au développement des nouvelles technologies de paiement (cartes à mémoire, opérations bancaires sur Internet, etc.). Une autre étude ${ }^{24}$ a été consacrée aux techniques de blanchiment de capitaux, qui a laissé apparaître un recours croissant à des institutions financières non bancaires (bureaux de change, sociétés de transfert de fonds et professions non financières).

En conclusion à leurs travaux, les membres du GAFI ont reconnu «absolument nécessaire de poursuivre l'action à l'échelle internationale pour approfondir et élargir la lutte contre le blanchiment de capitaux $»^{25}$. Cette question fera l'objet d'une attention particulière pendant l'année 1997-98, pendant laquelle sera étudiée l'évolution des activités, des structures et de la composition du GAFI.

21. Allemagne, Australie, Autriche, Belgique, Canada, Danemark, Espagne, Etats-Unis, Finlande, France, Grèce, Hongkong, Irlande, Islande, Italie, Japon, Luxembourg, Norvège, Nouvelle-Zélande, Portugal, Pays-Bas, Royaume-Uni, Singapour, Suède, Suisse et Turquie.

22. Commission européenne et Conseil de coopération du Golfe.

23. Groupe d'action financière sur le blanchiment d'argent de capitaux, Rapport annuel 1996-1997, GAFI-VIII, juin 1997. Disponible sur Internet: http://www. oecd. org/fatf/

24. Il s'agit du Rapport du GAFI-VIII sur les typologies du blanchiment de l'argent; disponible sur Internet: http://www. oecd. org/fatf/

25. Groupe d'action financière sur le blanchiment d'argent de capitaux, Rapport annuel 1996-1997, GAFI-VIII, juin 1997 , p. 28 


\section{$\square$ Fonds de dictateurs déposés en Suisse et entraide judiciaire}

De nombreuses affaires d'avoirs de dictateurs déposés auprès des banques suisses défrayent régulièrement la chronique depuis une vingtaine d'années. Bon nombre de dirigeants de pays en développement sont accusés d'avoir placé en Suisse des sommes d'argent importantes prélevées sur le patrimoine public de leur pays. Parmi les affaires les plus retentissantes, on peut citer celles du général paraguayen Stroessner, du Panaméen Noriega, du Philippin Ferdinand Marcos, de l'Haïtien Duvalier, du Zaïrois Mobutu Sese Seko ou encore du Malien Moussa Traoré. La première loi d'entraide en matière pénale à envisager le blocage des avoirs spoliés, sous de nombreuses conditions, est entrée en vigueur en 1983. Avant cette date, les demandes de rapatriement des fonds détournés et suspectés d'être dans des coffres suisses, n'étaient possibles que dans des cas très limités. Depuis 1983 la situation a évolué puisque, le $1^{\text {er }}$ février 1997, la révision de la loi d'entraide judiciaire, simplifiant la procédure, est entrée en vigueur.

L'entrée en vigueur de la nouvelle loi d'entraide judiciaire ainsi qu'une volonté politique d'en finir avec certains dossiers encombrants ont permis d'avancer sur quelques dossiers.

\section{Mali}

Pour la première fois, la Suisse a restitué à un pays, dont toutes les conditions de la procédure d'entraide ont été respectées, des fonds déposés dans les banques helvétiques. Ainsi la Suisse a remis aux autorités du Mali un montant de plus de 3,9 millions de francs détournés sous l'ancien gouvernement du président Moussa Traoré. Cette restitution a pu être réalisée grâce à l'Etat malien, qui s'est conformé à toutes les démarches exigées par l'entraide judiciaire internationale. La demande d'entraide judiciaire a été formellement présentée à la Suisse en 1991. Elle faisait suite à l'ouverture d'une procédure pénale au Mali pour détournement de deniers publics, intentée contre l'ex-président Traoré et d'autres responsables de l'ancien régime. A la demande du nouveau gouvernement malien, la DDC a pris en charge les honoraires du bureau d'avocats chargé de représenter le Mali dans la procédure d'entraide judiciaire.

\section{- Philippines}

Après douze ans de rebondissements, l'affaire des fonds Marcos a trouvé son dénouement judiciaire. Dans un premier arrêté rendu public le 12 décembre 1997, le Tribunal fédéral (TF) a ordonné le retour aux Philippines de 100 millions de dollars appartenant aux héritiers de Ferdinand Marcos et bloqués en Suisse depuis 1986. Mais il a approuvé le transfert des avoirs du président Marcos sur un compte bloqué auprès de la Banque nationale des Philippines à deux conditions. La première consiste en une garantie des Philippines que la confiscation et la restitution des avoirs feront l'objet d'une décision dans le cadre d'une procédure judiciaire conforme aux principes énoncés dans le Pacte international relatif aux droits civils et politiques. De plus, les Philippines doivent renseigner les autorités suisses sur la procédure judiciaire de confiscation et de restitution ainsi que sur les mesures prises pour dédommager les victimes de violations des droits de l'Homme sous le régime Marcos. En janvier 1998, le TF a rendu cinq nouveaux arrêts similaires portant sur des avoirs de plus de 420 millions de dollars. Dans chaque décision, le TF s'appuie sur la dernière révision de la loi d'entraide judiciaire. Celle-ci admet que, dans des cas exceptionnels, comme l'est l'affaire Marcos, le transfert de fonds à un Etat requérant n'est plus subordonné 
à un jugement exécutoire. Fin janvier 1998, l'ambassade des Philippines à Berne a fourni une garantie qui satisfait les conditions fixées par le $\mathrm{TF}^{26}$.

\section{- République démocratique du Congo (ex-Zaïre)}

Depuis quelques années des estimations circulent sur le montant de la fortune de l'ex-dictateur Mobutu Sese Seko. La plus souvent citée est celle émise par un ancien fonctionnaire du Fonds monétaire international, qui l'évaluait en 1990 à 4 milliards de francs. D'après le nouveau gouvernement de la République démocratique du Congo, les montants déposés en Suisse s'élèveraient à 11 milliards de dollars. Le 7 mai 1997, le gouvernement de Kabila a déposé une demande d'entraide judiciaire auprès de la Suisse afin de geler les avoirs de Mobutu et de lui remettre les documents y relatifs. A titre provisoire, l'Office fédéral de la police $(\mathrm{OFP})$ avait ordonné le blocage de sa villa à Savigny, estimée à 8 millions de francs, mais faute d'information complémentaire n'avait pas procédé au gel des avoirs. C'est suite à une décision du Conseil fédéral, le 17 mai, qu'une ordonnance a été édictée permettant de bloquer quelque 6 millions de francs. Suite au décès de Mobutu, les nouvelles autorités du Congo ont fait parvenir une autre demande d'entraide en septembre 1997, dans laquelle figurent les noms de Mobutu et de 59 de ses proches (ministres et responsables économiques). Début décembre 1997, l'OFP a annoncé qu'il avait partiellement donné suite à la demande d'entraide judiciaire. Dans sa décision d'entrée en matière, l'office constate que les conditions requises pour l'octroi de l'entraide judiciaire sont remplies, ordonne le gel d'autres comptes et charge le Cabinet des juges d'instruction genevois de recueillir les documents bancaires concernés. Le blocage de la villa de Savigny est maintenu jusqu'à la clôture de la procédure d'entraide ${ }^{27}$. Certaines ONG (Déclaration de Berne, Action Place financière suisse et Communauté de travail) ont, à plusieurs reprises, demandé au Conseil fédéral de geler les avoirs de Mobutu en Suisse ${ }^{28}$.

\section{- Pakistan}

En octobre 1997, l'Office fédéral de la police a accordé l'entraide judiciaire aux autorités pakistanaises et a chargé la police genevoise d'instruire les huit cas de corruption dénoncés par le Parquet général du Pakistan à l'encontre de l'ancien premier ministre Benazir Bhutto et d'Asif Ali Zardari, son époux. Quelque 20 millions de francs placés sur les comptes de Benazir Bhutto et de ses proches ont été bloqués ${ }^{29}$.

ASTM 1997, révision de la loi sur l'entraide judiciaire, p. 98.

\section{$\square$ Convention de l'OCDE contre la corruption}

«Actuellement, la corruption suit malheureusement le rythme de la globalisation. Ce phénomène fausse non seulement la concurrence, mais il compromet aussi l'efficacité des politiques de développement. A l'heure du marché global, les entreprises doivent pouvoir compter sur des règles de jeu loyales. L'engagement

26. Office fédéral de la police, Communiqué de presse, 26 janvier 1998, «Restitution des fonds Marcos».

27. Office fédéral de la police, Communiqué de presse, $1^{\text {er }}$ décembre 1997, «Entraide judiciaire dans l'affaire Mobutu: l'OFP a arrêté une décision d'entrée en matière».

28. Déclaration de Berne, Action Place financière suisse, Communiqué de presse, 14 mai 1997, «Le Conseil fédéral doit geler immédiatement les avoirs de Mobutu en Suisse», et Communauté de travail, «Prise de position 1997 ».

29. Office fédéral de la police, Communiqués de presse, 15 septembre 1997 «Comptes de Benazir Bhutto bloqués à titre provisoire», 15 octobre 1997 «Comptes de Benazir Bhutto bloqués: 20 millions bloqués». 
de recourir à la sanction pénale est d'une importance capitale. $»^{30}$ C'est en ces termes que s'est exprimé Jean-Pascal Delamuraz lors de la signature d'une convention de l'OCDE à Paris le 17 décembre 1997. Cette convention vise l'interdiction de versement de pots-de-vin par des entreprises afin de remporter des marchés.

Le 20 novembre 1997, les pays membres de l'OCDE, dont la Suisse, et cinq pays non membres, l'Argentine, le Brésil, la Bulgarie, le Chili et la République slovaque, ont adopté une Convention sur la lutte contre la corruption d'agents publics étrangers dans les transactions commerciales internationales ${ }^{31}$. Les EtatsUnis, qui ont une loi anticorruption depuis 1977, ont joué un rôle déterminant dans ces négociations. Cette convention, aboutissement de deux ans de négociations, prévoit pour les Etats parties de faire de la corruption de fonctionnaires étrangers un délit pénal. Le texte demande que des mesures efficaces soient prises pour «décourager, prévenir et combattre» la corruption de fonctionnaires étrangers dans le cadre de négociations commerciales. Afin de rendre cet instrument opérationnel, chaque Etat partie à la convention devra adapter son système juridique afin de pouvoir appliquer des sanctions pénales aux personnes physiques, sanctions pouvant aller jusqu'à des peines de prison. La convention s'est également dotée de dispositions pouvant être appliquées aux entreprises. Le texte souligne que «si dans un système juridique d'une partie, la responsabilité pénale n'est pas applicable aux personnes morales, cette Partie fait en sorte que les personnes morales soient passibles de sanctions non pénales efficaces, proportionnées et dissuasives, y compris pécuniaires, en cas de corruption d'agents publics étrangers». La convention aborde également la question de la coopération internationale et de la surveillance de la mise en œuvre du texte.

\section{Position de la Suisse}

La Suisse a participé activement à l'élaboration du texte: le professeur bâlois Mark Pieth a dirigé le groupe d'experts chargé des travaux préparatoires. Par ailleurs l'ambassadeur Marino Baldi (OFAEE) a présidé la conférence de négociation. La Convention, signée par la Suisse, devra être ratifiée par le Parlement; la discussion est prévue aux Chambres pour le deuxième semestre de 1998.

\section{LE CONSEIL FÉDÉRAL VEUT SUPPRIMER LA DÉDUCTIBILITÉ FISCALE DES «POTS-DE-VIN》}

D EPIS de nombreuses années, la Suisse autorise une pratique de plus en plus contestée. En effet, entreprises et hommes d'affaires helvétiques ont le droit de payer des dessous-de-table à des fonctionnaires étrangers pour décrocher des nouveaux marchés, puis de les déduire de leur déclaration d'impôts en les faisant passer comme "frais d'acquisition». Cette pratique, qui vise surtout à faciliter l'obtention de contrats dans les pays en développement, a été légitimée en 1946 par le Tribunal fédéral, puis confirmée par une circulaire de l'Administration fédérale des contributions.

A plusieurs reprises des parlementaires ont tenté de mettre un terme à cette pratique. Mais il aura fallu attendre mars 1995 pour qu'une initiative parlementaire du conseiller national tessinois Werner

30. L'Agefi, 18 décembre 1997 «Lutte contre la corruption».

31. Le texte de la convention sur la lutte contre la corruption, ainsi que les commentaires s'y rapportant, sont accessibles sur le site Internet de l'OCDE: http://www. OECD. ORG/daf/cmis/bribery/20novlf. htm 
Carobbio (PS) soit acceptée de justesse par le Conseil national. L'initiative Carobbio propose d'interdire la déduction fiscale des pots-de-vin versés à des fonctionnaires, aussi bien suisses qu'étrangers. Cette initiative, qui devra encore obtenir l'aval du Parlement en 1998, a d'ores et déjà été acceptée par le Conseil fédéral. Par cette acceptation, le gouvernement suit la recommandation positive formulée par la Commission de l'économie et des redevances du Conseil national.

En octobre 1997, le Conseil fédéral a condamné le versement de pots-de-vin, précisant que la corruption mine la confiance dans l'administration et dans l'Etat. Et de préciser que la Suisse n'admettra plus désormais la corruption de fonctionnaires étrangers. Pour ce faire, le gouvernement entend modifier le Code pénal suisse (les travaux ont déjà commencé à cet effet), ainsi que l'article 27 de la loi fédérale sur l'impôt fédéral direct. L'évolution de la position suisse s'inscrit dans un contexte international où le thème de la corruption est devenu très sensible, comme en témoigne la récente convention de l'OCDE contre la corruption signée en décembre 1997. La révision du Code pénal et de la loi sur l'impôt fédéral direct ainsi que la ratification de la convention de l'OCDE seront à l'ordre du jour des Chambres fédérales en 1998.

Sources: Journal de Genève, 23 octobre 1997 "Le Conseil fédéral veut déclarer la guerre aux dessous-de-table officiels". La Liberté, 23 octobre 1997 "Un pas de plus a été franchi dans la lutte contre la corruption».

\section{$\square$ Engagement du FMI et de la Banque mondiale contre la corruption}

Lors de leur réunion annuelle conjointe, qui s'est tenue à Hongkong en septembre 1997, le Fonds monétaire international et la Banque mondiale ont réaffirmé publiquement et conjointement leur engagement dans la lutte contre la corruption. Les institutions vont renforcer les contrôles sur le plan interne au sein de leurs propres projets. Elles ont également annoncé qu'elles prendraient en compte «la mauvaise gestion des affaires publiques» dans l'attribution de leur assistance à leurs pays membres. A titre d'exemple, en août 1997, le FMI avait refusé une aide de 215 millions de dollars au Kenya, considérant que la destination finale des fonds ne pouvait être assurée.

Dans le Rapport sur le développement dans le monde $1997^{32}$ de la Banque mondiale, un chapitre est consacré à la corruption. Sous le titre «Mettre fin à l'arbitraire et à la corruption», la banque invite chaque pays «à mettre en place et à consolider des mécanismes qui donneront aux organes de l'Etat la souplesse et les incitations voulues pour agir dans l'intérêt de tous, mais qui, parallèlement, feront barrière à l'arbitraire et à la corruption dans leurs rapports avec les entreprises et les citoyens». Le rapport passe également en revue les mécanismes visant à endiguer la corruption.

Pour sa part, le Conseil exécutif du Fonds monétaire international a adopté des directives destinées à renforcer son rôle de promoteur des principes dit de good governance. Un document ${ }^{33}$ détaille ces nouvelles règles qui visent à lutter contre la corruption et à garantir un secteur public responsable de ses dépenses.

32. Banque mondiale, L'Etat dans un monde en mutation - Rapport sur le développement dans le monde 1997, Washington, Banque mondiale, 1997.

33. Fonds monétaire international, Guidance Note, «The Role of the IMF in Governance Issue», July 1997. Disponible sur Internet: http://www. imf. org 


\section{$\square$ Déclaration de Lima}

A l'invitation de Transparency International, et sous le patronage du gouvernement péruvien, s'est tenue à Lima en septembre 1997 la $8^{\mathrm{e}}$ Conférence internationale anticorruption. Plus de 1000 délégués venus de 93 pays, parmi lesquels des ministres, des hauts fonctionnaires (des représentants de la DDC pour la Suisse), des membres d'associations professionnelles, des procureurs généraux (dont Carla Del Ponte), des juges, des avocats, des députés, des journalistes et des représentants d'ONG, étaient présents. Certaines organisations internationales (ONU, Banque mondiale, FMI, Organisation des Etats américains, OCDE, Conseil de l'Europe, Interpol, Banque interaméricaine de développement) étaient représentées par des fonctionnaires internationaux.

A l'issue de la conférence, les délégués ont adopté la Déclaration de Lima contre la corruption ${ }^{34}$ qui appelle les gouvernements, les organisations internationales et régionales ainsi que les citoyens à mobiliser leurs efforts et leurs énergies afin de lutter contre la corruption.

\section{SOURCES}

Blanchiment d'argent

Loi sur le blanchiment d'argent du 10 octobre 1997, in Feuille fédérale, n 41 , vol. IV, 21 octobre 1997 (message 96.055 ).

"Message relatif à la loi fédérale concernant la lutte contre le blanchissage d'argent dans le secteur financier», in Feuille fédérale, $\mathrm{n}^{\circ} 37$, vol. III, 17 septembre 1996.

Bulletin officiel de l'Assemblée fédérale - Conseil national et Bulletin officiel de l'Assemblée fédérale - Conseil des Etats, sessions de 1997.

Groupe d'action financière sur le blanchiment d'argent de capitaux, Rapport annuel 1996-1997, GAFI-VIII, juin 1997.

Stephan Trechsel (Hrsg.), Geldwäscherei - Prävention und Massnahmen zur Bekämpfung, Zürich, Schulthess Polygraphischer Verlag, 1997.

L'Agefi, 18 mars 1997 «L'étude de la loi sur le blanchiment combattue sur un seul point», 8 octobre 1997 «La loi sur le blanchiment est sous toit».

La Liberté, 26 septembre 1997 «Le blanchiment par négligence sera puni aussi chez les intermédiaires» et «Une seule divergence sur le blanchiment».

Neue Zürcher Zeitung, 28. Dezember 1996 «Mit Meldepflicht und Vermögenssperre gegen die Geldwäsche», 20. Juni 1997 «Technisch überholter Kampf gegen Geldwäscherei».

Fuite de capitaux et entraide judiciaire

Office fédéral de la police, Communiqués de presse, 1997 et 1998.

Déclaration de Berne, Action Place financière suisse, Communiqué de presse, 14 mai 1997, «Le Conseil fédéral doit geler immédiatement les avoirs de Mobutu en Suisse».

Communauté de travail, «Prise de position 1997».

L'Hebdo, 3 avril 1997 «L'argent des dictateurs a de l'odeur».

L'Agefi, 15 décembre 1997 «Le TF ouvre une brèche pour les fonds Marcos», 16 janvier 1998 «Les fonds Marcos seront restitués».

Journal de Genève, 9 septembre 1997 «Mobutu mort, saura-t-on enfin combien il a laissé d'argent en Suisse?», 20 septembre 1997 «Pour récupérer les avoirs de Mobutu, la Suisse veut un interlocuteur au Congo», 19 janvier 1998 (affaire Bhutto),

La Liberté, 25 mars 1997 «La Suisse va rendre 3,2 millions au Mali», 20 septembre 1997 «La Suisse a remis 3,9 millions au Mali». 
Corruption

Fonds monétaire international, FMI Bulletin, «La corruption liée aux investissements risque de freiner la croissance», 8 décembre 1997.

Déclaration de Berne, Vers un développement solidaire, dossier «Corruption», n¹37, janvier 1997.

Transparency International, Déclaration de Lima contre la corruption, septembre 1997.

L'Agefi, 27 mai 1997 «Les pays industrialisés s'engagent sur un futur pacte anti-corruption», 18 décembre 1997 «Lutte contre la corruption, la Suisse s'y met aussi».

Journal de Genève, 9 février 1997 «La place financière se barricade contre les capitaux du crime», 28 mai 1997 «L'OCDE planche sur les mesures de lutte contre la corruption», 5 août 1997 «Le FMI ne veut plus financer un Kenya corrompu», 6 août 1997 «Le FMI veut lutter contre la corruption», 25 septembre 1997 «La Banque mondiale part en guerre contre la corruption», 8 octobre 1997 «Quand la corruption atteint les plus hautes sphères», 18 décembre 1997 «A Paris, 35 Etats signent une convention contre la corruption».

Neue Zürcher Zeitung, 28. Januar 1997 «Der Korruptionsbericht - Ein Beruhigungsmittel?», 27. Mai 1997 «Aufbruch zu neuen Ufern am OECD-Ministerrat», 13. September 1997 «Aufruf zum weltweiten Kampf gegen Korruption», 23. September 1997 «Korruptionsbekämpfung und Schuldenerlass», 18. November 1997 «Die OECD im Kampf gegen die Korruption», 22. November 1997 «Eine Konvention gegen die Korruption».

\section{SITES INTERNET}

GAFI (Groupe d'action financière sur le blanchiment de capitaux de l'OCDE). Le site contient des copies de documents officiels (notamment le texte des «quarante recommandations») ainsi que le résumé des travaux du GAFI, son programme de travail, sa composition et ses liens avec d'autres organisations internationales. http://www. oecd. org/fatf/

Transparency International (TI). Créée en 1993, TI est une organisation non gouvernementale qui lutte contre la corruption dans les transactions commerciales. Le site contient une description des objectifs et des activités de l'organisation, des liens avec les différentes sections nationales de TI (dont la section suisse), ses communiqués de presse et publications, ainsi qu'une liste des conférences officielles ou non ayant pour sujet la corruption. http://www. transparency. de

\subsection{COMMERCE DES ARMES}

Au cours de l'année 1996, le commerce des armes au niveau mondial a subi une légère régression par rapport à l'année précédente. A noter que depuis dix ans, les ventes d'armements ont diminué de moitié dans le monde; les exportations d'armes suisses ont suivi la même tendance. 1997 aura été marqué par la conclusion d'un traité international visant à interdire la fabrication, le commerce et l'utilisation des mines antipersonnel. Grâce à la pression de l'opinion publique internationale, plus de 122 Etats ont signé le traité lors d'une conférence à Ottawa en décembre 1997. Sur le plan suisse une initiative populaire visant à interdire les exportations de matériel militaire a été rejetée par le peuple et les cantons en juin 1997.

\section{$\square$ Commerce international des armes}

En 1996, les ventes d'armes ont totalisé 22,98 milliards de dollars dans le monde, en légère régression par rapport à 1995 (23,2 milliards), selon le rapport du SIPRI $^{35}$. Si les Etats-Unis demeurent le principal exportateur, leur part dans les ventes mondiales a continué à baisser à 44\% en 1996 (contre 47\% en 1995 et $55 \%$ en 1994), alors que la Russie se repositionne comme un important fournisseur d'armements après la période de turbulences qui a suivi la dissolution de l'URSS. En un an, la Russie a accru sa part de $15 \%$ à $20 \%$. Le rapport souligne également la part croissante des exportations d'armes des pays de l'Union européenne, qui a atteint un nouveau record de $28 \%$ en 1996. Les ventes d'armes dans le monde ont diminué de moitié, de 44 à 23 milliards de dollars entre 1987 et 1996, mais celles de l'UE ont à peine baissé, passant de 6,8 à 6,4 milliards de dollars pendant la même période.

35. Stockholm International Peace Research Institute, Yearbook 1997, SIPRI, June 1997. Version électronique du rapport SIPRI 1997: http://www. sipri. se/pubs/yearb. html 
L'Asie a été le principal acheteur d'armes avec une part de $48 \%$ des importations mondiales. Près d'un tiers de ces importations concernent la Chine, la Corée du Sud et Taiwan. Le Moyen Orient, naguère le principal marché dans le monde, a maintenu sa part à environ $25 \%$ des importations mondiales.

\section{Exportations suisses d'armes}

Les exportations suisses de matériel de guerre ont à nouveau augmenté l'an dernier, passant de 141,2 à 232,9 millions de francs (tableau n ${ }^{\circ} 17$ ). Leur proportion face à l'ensemble des marchandises suisses exportées, passe de $0,15 \%$ à $0,23 \%$, alors que les exportations de matériel de guerre ne cesșaient de décliner depuis 1987.

\section{Tableau $\mathbf{n}^{\circ} 17$}

Exportations suisses de matériel de guerre 1995-1996

Les 15 principaux pays en développement et en transition clients de la Suisse

\begin{tabular}{|c|c|c|c|c|c|}
\hline \multicolumn{3}{|c|}{1995} & \multicolumn{3}{|c|}{1996} \\
\hline Pays $^{1}$ & $1000 \mathrm{fr}$. & en \% & Pays $^{1}$ & $1000 \mathrm{fr}$. & en $\%$ \\
\hline Malaisie & 6558 & 4.6 & Oman & 65426 & 28.1 \\
\hline Inde & 5318 & 3.8 & Inde & 5997 & 2.6 \\
\hline Singapour & 4345 & 3.1 & Singapour & 5862 & 2.5 \\
\hline Brésil & 2129 & 1.5 & Arabie Saoudite & 5472 & 2.3 \\
\hline Emirats arabes & 2090 & 1.5 & Afrique du Sud & 3762 & 1.6 \\
\hline Argentine & 1920 & 1.4 & Pakistan & 2927 & 1.3 \\
\hline Corée du Sud & 1102 & 0.8 & Brésil & 2489 & 1.1 \\
\hline Pakistan & 648 & 0.5 & Emirats arabes & 2398 & 1.0 \\
\hline Maroc & 415 & 0.3 & Bahreïn & 1412 & 0.6 \\
\hline Tunisie & 297 & 0.2 & Thaïlande & 1262 & 0.5 \\
\hline Bulgarie & 190 & 0.1 & Malaisie & 908 & 0.4 \\
\hline Egypte & 133 & 0.1 & Egypte & 757 & 0.3 \\
\hline Bahreïn & 121 & 0.1 & Corée du Sud & 735 & 0.3 \\
\hline Croatie & 120 & 0.1 & Tunisie & 704 & 0.3 \\
\hline Oman & 96 & 0.1 & Israël & 678 & 0.3 \\
\hline $\begin{array}{l}\text { Autres pays en dévelop } \\
\text { pement et en transition }\end{array}$ & 1256 & 0.9 & $\begin{array}{l}\text { Autres pays en dévelop } \\
\text { pement et en transition }\end{array}$ & p- 3565 & 1.5 \\
\hline $\begin{array}{l}\text { Total pays en dévelop- } \\
\text { pement et en transition }\end{array}$ & 26739 & 18.9 & $\begin{array}{l}\text { Total pays en dévelop- } \\
\text { pement et en transition }\end{array}$ & 104354 & 44.8 \\
\hline Pays industrialisés & 114429 & 81.1 & Pays industrialisés & 128584 & 55.2 \\
\hline Exportations totales & 141169 & 100.0 & Exportations totales & 232938 & 100.0 \\
\hline
\end{tabular}

1. Pays de destination finale.

Source: Département militaire fédéral. 
A noter que le total des exportations suisses de matériel de guerre vers les pays en développement a presque quadruplé entre 1995 et 1996, passant de 26,7 millions de francs à plus de 104,3 millions de francs. Cette augmentation s'explique par une commande exceptionnelle d'Oman pour un montant de 65 millions de francs suisses, mais également par des commandes plus importantes en 1996 de certains pays en développement (Afrique du Sud, Pakistan, etc.).

Le Comité pour le désarmement et l'arrêt des exportations d'armes (ARW) s'est montré très critique à l'égard des ventes à Oman, mais également à l'Arabie Saoudite, à l'Inde et au Pakistan. L'organisation rappelle qu'au sens de la loi de 1973 sur le matériel de guerre il est interdit de livrer des armes dans les zones dangereuses en situation de tension ou de guerre, dans les pays qui méprisent les droits de l'Homme et dans les régions où elles compromettent les efforts de la coopération suisse au développement ${ }^{36}$.

\section{$\square$ Mines antipersonnel}

Depuis 1992, une dizaine d'organisations non gouvernementales à travers le monde (elles sont plus de 1000 aujourd'hui!) se sont mobilisées pour convaincre les responsables gouvernementaux de la nécessité d'interdire totalement et immédiatement la fabrication, la commercialisation et l'utilisation des mines antipersonnel. Cette campagne internationale a permis de sensibiliser l'opinion publique mondiale émue et choquée par les conséquences de la prolifération des mines sur les populations civiles. Sous la pression populaire et de certains pays (Canada notamment), les Etats ont conduit des négociations dans un temps record qui ont abouti à la signature d'un traité visant l'interdiction totale des mines.

Signature d'un traité visant l'interdiction totale des mines antipersonnel

Le processus engagé en octobre 1996 à Ottawa devant conduire à l'interdiction totale des mines antipersonnel s'est poursuivi en 1997, année pendant laquelle les Etats se sont réunis à trois reprises.

- Conférence de Bruxelles, juin 1997

La Conférence de Bruxelles a réuni 130 Etats qui ont débattu sur l'opportunité de négocier un traité sur l'interdiction totale des mines antipersonnel; 97 pays, soit la moitié de la communauté internationale, se sont engagés à négocier lors d'une prochaine conférence, fixée pour le mois de septembre suivant à Oslo, le texte d'un traité dont l'objectif d'interdiction totale (fabrication, commercialisation et utilisation) était clairement affiché. Une ombre au tableau de cette conférence; l'absence de pays producteurs comme la Chine ou l'Inde.

\section{- Conférence d'Oslo, septembre 1997}

C'est lors de la Conférence d'Oslo que s'est négocié le texte du traité, dont l'objectif d'interdiction totale a été clairement défini et validé par plus de 100 Etats. Les négociations du traité ont porté sur les points suivants:

- l'interdiction complète de l'emploi, du stockage, de la production et du transfert de mines terrestres antipersonnel;

- la destruction des mines, qu'elles proviennent des stocks ou du déminage; 
- la coopération et l'assistance internationales en matière de déminage et d'aide aux victimes dans les pays affectés.

Deux problèmes ont persisté lors de cette conférence: l'absence répétée de pays producteurs comme la Chine et l'Inde, d'une part; l'attitude des Etats-Unis, d'autre part, qui par leurs interventions ont cherché à vider le traité de toute substance. Leur retrait de la négociation a cependant permis de sauver les objectifs du traité.

Au terme de cette conférence, les représentants des 89 pays présents ont adopté formellement le texte du traité, rejetant toutes les demandes américaines.

- Conférence d'Ottawa, décembre 1997

Cent vingt-deux Etats ont signé le traité d'interdiction totale des mines antipersonnel sur les 156 présents lors de la Conférence d'Ottawa. Les pays signataires s'engagent à détruire leurs stocks de mines, à déminer leurs territoires et à venir en aide aux victimes. Certes, la signature de ce traité est une réussite incontestable, mais n'est qu'un premier pas en avant; 18 pays présents à Ottawa n'ont pas signé le traité, dont les Etats-Unis, la Chine, la Russie, ainsi que des pays en situation de conflit (les deux Corée, Irak, Iran, Pakistan, Inde, Israël, Serbie, etc.). L'universalité du traité est donc loin d'être acquise. Les différents Etats doivent maintenant procéder au dépôt des instruments de ratification; le traité d'Ottawa n'entrera en vigueur qu'après avoir été ratifié par 40 pays. La délégation suisse était conduite par le conseiller fédéral Flavio Cotti, qui a signé, au nom de la Suisse, le nouveau traité.

\section{Position suisse \\ La Suisse a participé activement au processus d'Ottawa en vue de l'interdiction des mines antipersonnel. A son initiative, un noyau informel d'Etats ${ }^{37} \mathrm{~s}^{\text {'est }}$ constitué en février 1997 pour accompagner et influencer le processus de manière discrète, mais constante. La Suisse s'est engagée pour une interdiction totale de l'emploi, de la production, du stockage et du transfert des mines anti- personnel, pour le déminage obligatoire ainsi que pour la destruction des stocks. Lors des négociations la Suisse a veillé à ce qu'aucune concession essentielle ne soit faite dans les domaines clés de la convention ${ }^{38}$. Son objectif premier était d'obtenir un traité ambitieux et contraignant plutôt que d'aboutir à un texte faible qui aurait réuni un consensus plus large.}

Depuis le lancement de la Campagne suisse contre les mines antipersonnel en 1992, la position du Département militaire fédéral a évolué vers une attitude plus ferme pour une interdiction des mines. Le succès de la campagne rencontré auprès du public suisse ainsi que la décision du Parlement de décembre 1996 d'inclure les mines antipersonnel dans la loi interdisant les armes nucléaires, biologiques et chimiques expliquent en partie cette évolution.

ASTM 1997, pp. 108 et 109.

Sur le plan interne, la Suisse a pris les mesures nécessaires afin de remplir les conditions pour pouvoir signer et ratifier la convention d'Ottawa. En octobre

37. Faisaient partie de ce noyau informel: Afrique du Sud, Allemagne, Autriche, Belgique, Canada, Irlande, Mexique, Norvège et Nouvelle-Zélande.

38. Département fédéral des affaires étrangères, Communiqué de presse, Berne, 26 novembre 1997, «Convention sur l'interdiction des mines antipersonnel». 
1995, le Département militaire fédéral avait renoncé à utiliser, et a donc détruit, ses stocks de mines antipersonnels «traditionnels». Cependant, en 1994 et 1995, elle avait acheté d'importantes quantités de mines à double usage (déclenchement suite à un contact et déclenchement à distance). Afin que ces armes soient compatibles avec la convention d'Ottawa, la Suisse a supprimé le dispositif de déclenchement automatique et a conservé la totalité du stock des mines à double usage, ce qu'a regretté la Campagne suisse des mines antipersonnel ${ }^{39}$.

D'autre part, en novembre 1997, le Conseil fédéral a décidé de créer un Centre international de déminage humanitaire (CIDH). Le centre, qui sera constitué en fondation, devra favoriser la coordination des opérations de déminage sur le terrain par le biais de conférences internationales et la création de banques de données informatiques. Dès 2001, le CIDH formera des spécialistes en technique de destruction de mines. Sur le plan financier, ce projet coûtera 2,25 millions de francs par an à la Confédération les premières années, puis 5,5 millions de francs à partir de 2001. Le centre sera amené à collaborer avec le CICR et l'ONU. Plusieurs pays, parmi lesquels la Grande-Bretagne, les Etats-Unis, la France et la Suède, ont déjà manifesté leur intérêt pour participer à ces opérations.

\section{Prix Nobel pour la Campagne internationale contre les mines}

Le prix Nobel de la paix 1997 a été décerné collectivement aux 1000 associations regroupées au sein de la Campagne internationale pour l'interdiction des mines antipersonnel. Ce prix est l'expression du rôle joué par les associations de la campagne et démontre le potentiel de mobilisation et de pression de l'opinion publique mondiale. Le prix a été remis le 10 décembre 1997 à Oslo à Jody Williams, coordinatrice de la campagne internationale, et à Tun Channareth, victime d'une mine. A noter également l'engagement du Comité international de la Croix-Rouge, qui dès les débuts de la campagne s'est fortement engagé pour la réussite du processus.

La Campagne suisse contre les mines antipersonnel regroupe une quarantaine d'associations. En septembre 1995, elle avait déposé à Berne une pétition forte de 150'000 signatures réclamant aux Etats d'interdire totalement la production, la commercialisation et l'usage de mines et à la Suisse de se prononcer officiellement en faveur de cette interdiction. Elisabeth Reusse-Decrey, coordinatrice de la campagne en Suisse, a accompagné les délégations officielles lors des conférences internationales.

\section{$\square$ Initiative "pour l'interdiction d'exporter du matériel de guerre»}

En juin 1997, le peuple suisse s'est prononcé sur l'initiative populaire «pour l'interdiction d'exporter du matériel de guerre». Cette initiative avait été déposée par le Parti socialiste suisse en 1992. Elle exigeait une interdiction totale des exportations de matériel de guerre et entendait également interdire l'exportation de produits à double usage, civil et militaire, dès lors que ces produits étaient destinés à des buts militaires. En outre, elle exigeait de la Confédération que celle-ci s'engage davantage au niveau international pour limiter le commerce d'armements dans le monde. 
Dans son message ${ }^{40}$ concernant l'initiative, le Conseil fédéral avait recommandé son rejet, suivi par le Parlement ${ }^{41}$. Lors de la campagne d'information sur les enjeux de la votation, deux fronts très marqués sont apparus entre les opposants à l'initiative (les partis de droite, l'industrie des machines, le Vorort) et les supporters (le Parti socialiste suisse, le GSSA, les mouvements de paix et les organisations de développement). Certaines «défections» sont cependant apparues parmi les organisations ayant soutenu l'initiative lors de son lancement. Les syndicats (Union syndicale suisse et FTMH) se sont distancés du Parti socialiste, laissant la liberté de vote. Les œuvres d'entraide catholiques (Caritas, Action de Carême et Missio) n'ont pas formulé de consigne de vote. Cependant, elles ont publié ensemble une brochure, Investir dans le développement humain ${ }^{42}$, mettant l'accent sur les questions éthiques et de politique de développement devant être prises en compte dans le débat sur les exportations d'armes. Mais le débat s'est surtout concentré sur les risques de perte d'emplois ${ }^{43}$, laissant peu d'espace aux arguments éthiques ou aux choix de société.

Le peuple (à $77,3 \%$ ) et tous les cantons ont rejeté l'initiative lors de la votation du 8 juin. Par ce rejet, le peuple a donné sa préférence à la loi révisée sur le matériel de guerre et à la loi sur le contrôle des biens, proposées par le Conseil fédéral comme contre-projet.

@ ASTM 1997, «Révision de la loi fédérale sur le matériel de guerre», p. 105.

\section{$\square$ Dépôt d'une nouvelle initiative}

Le 26 mars 1997, l'initiative «Economiser dans l'armée et la défense générale Pour davantage de paix et d'emplois d'avenir» a été déposée auprès de la Chancellerie fédérale, munie de $115^{\prime} 000$ signatures.

Les auteurs de l'initiative considèrent qu'en cette fin de $X^{e}$ siècle, la Suisse, entourée de démocraties stables, ne peut plus se permettre le luxe de dépenser annuellement 6 milliards de francs pour l'armée. Ainsi, l'initiative populaire demande de réduire de moitié le budget militaire de la Confédération d'ici dix ans. Cette réduction de moitié dégagerait un dividende pour la paix de 3 milliards de francs par an à partir de 2006. Le texte demande la création d'un fonds de 1 milliard de francs pour promouvoir la conversion d'emplois militaires en emplois civils. Un tiers du montant économisé doit être affecté au renforcement de la politique de paix sur le plan international. Le reste doit être investi dans des projets sociaux (introduction d'une assurance maternité, lutte contre le chômage, renforcement de l'AVS).

L'initiative a été lancée en septembre 1995 par le Parti socialiste suisse, les Verts et des organisations pacifistes et de développement. Elle est la réponse à l'invalidation en 1995 par le Parlement de l'initiative «pour moins de dépenses militaires et davantage de politique de paix».

40. «Message concernant l'initiative populaire "pour l'interdiction d'exporter du matériel de guerre" et pour la révision de la loi fédérale sur le matériel de guerre", 15 février 1995, message 95.015.

41. Cette initiative a été rejetée par 111 voix contre 60 au Conseil national et par 32 voix contre 3 au Conseil des Etats.

42. Caritas, Missio, Action de Carême, Investir dans le développement humain-Aspects de politiques et éthiques de l'exportation d'armes, Lucerne, 1997. A commander auprès de Caritas Suisse, tél. 041/419.22.22.

43. Les opposants à l'initiative menaçaient d'une perte de 120'000 postes de travail, alors que les initiants évaluaient cette perte à 2000 emplois en cas d'acceptation de l'initiative. 


\section{SOURCES}

Stockholm International Peace Research Institute, Yearbook 1997 - Armement, Disarmement and International Security, SIPRI, June 1997.

Caritas, Missio, Action de Carême, Investir dans le développement humain-Aspects de politiques et éthiques de l'exportation d'armes, Lucerne, 1997.

Comité romand des initiatives pour la paix et le désarmement, Arguments pour ou contre l'interdiction d'exporter du matériel de guerre, 1997.

Comité suisse contre l'initiative anti-exportations, Non à l'initiative pour l'interdiction d'exporter du matériel de guerre, argumentaire et information brève, avril 1997.

Objectif Paix, journal du comité romand des initiatives pour la paix, parutions de 1997.

Déclaration de Berne, Vers un développement solidaire, "Votation pour une interdiction des exportations d'armes», $\mathrm{n}^{\circ} 139$, mai 1997.

L'Agefi, 27 juin 1997 «Le commerce international des armes en légère baisse».

Journal de Genève, 27 mars 1997 «Dépôt de l'initiative sur les dépenses militaires», 26 juin 1997 «La Conférence de Bruxelles sur les mines va vers un succès».

La Liberté, 6 février 1997 «Les exportations d'armes exécutent un redressement spectaculaire», 9 juin 1997 «Le souverain balaie l'initiative de la gauche sur les exportations d'armes», 19 septembre 1997 «Ottawa est une victoire des petits et des victimes sur les puissants», 12 octobre 1997 «Le jury d'Oslo met à l'honneur la lutte contre les mines antipersonnel», 27 novembre 1997 «Un Centre international de déminage humanitaire va être créé à Genève», 29 novembre 1997 «La convention d'interdiction totale va entrer en vigueur».

Mosquito, «Kriegs-Material-Exporte, Schutz der Wirtschaft oder Schutz der Menschenrechte?», Nr. 1, Februar 1997.

Nouveau Quotidien, 18 septembre 1997 «Un traité international va enfin interdire les mines antipersonnel».

Neue Zürcher Zeitung, 23 Juni 1997 «Bemühungen ein Antipersonenminen-Verbot», 21. August 1997 «Ist ein Totalverbot von Personenminen in Sicht?», 2. September 1997 «Suche nach Konsens zum Verbot von Landminen», 16. September 1997 «Vor einem umfassenden Personenminenverbot», 18. September 1997 «Personenminen-Verbot ohne Beteiligung der USA», 19. September 1997 «Ein Schritt zur Ächtung der Personenminen», 1. Oktober 1997 «Exportkontrollen für konventionelle Waffen», 11. Oktober 1997 «Ehrung der Kampagne gegen Landminen», 27. November 1997 «Genfer Zentrum für Minenräumung», 5. Dezember 1997 «Umfassende Ächtung der Personenminen».

\section{SITES INTERNET}

Institut international de recherche sur la paix (SIPRI): http://www. sipri. se/ Handicap International: http://www. handicap-international. org 